Article

\title{
On the Formation and Accumulation of Solid Carbon Particles in High-Enthalpy Flows Mimicking Re-Entry in the Titan Atmosphere
}

\author{
Antonio Esposito ${ }^{1}$, Marcello Lappa ${ }^{2, *}{ }^{\circledR}$, Gennaro Zuppardi ${ }^{1}$, Christophe Allouis ${ }^{3}(\mathbb{D}$,

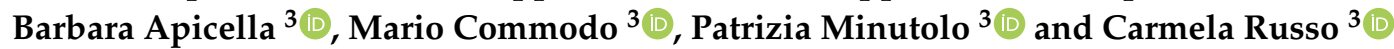 \\ 1 Department of Industrial Engineering, Aerospace Branch, University of Naples "Federico II" Via Claudio 21, \\ 80125 Naples, Italy; antespos@unina.it (A.E.); zuppardi@unina.it (G.Z.) \\ 2 Department of Mechanical and Aerospace Engineering, University of Strathclyde, James Weir Building, \\ 75 Montrose Street, Glasgow G1 1XJ, UK \\ 3 Istituto di Ricerche sulla Combustione, CNR, Piazzale Tecchio 80, 80125 Naples, Italy; allouis@irc.cnr.it (C.A.); \\ b.apicella@irc.cnr.it (B.A.); m.commodo@irc.cnr.it (M.C.); p.minutolo@irc.cnr.it (P.M.); c.russo@irc.cnr.it (C.R.) \\ * Correspondence: marcello.lappa@strath.ac.uk
}

Received: 10 March 2020; Accepted: 10 June 2020; Published: 12 June 2020

check for updates

\begin{abstract}
The problem relating to the formation of solid particles enabled by hypersonic re-entry in methane-containing atmospheres (such as that of Titan) has been tackled in the framework of a combined experimental-numerical approach implemented via a three-level analysis hierarchy. First experimental tests have been conducted using a wind tunnel driven by an industrial arc-heated facility operating with nitrogen as working gas (the SPES, i.e., the Small Planetary Entry Simulator). The formation of solid phases as a result of the complex chemical reactions established in such conditions has been detected and quantitatively measured with high accuracy. In a second stage of the study, insights into the related formation process have been obtained by using multispecies models relying on the NASA CEA code and the Direct Simulation Monte Carlo (DSMC) method. Through this approach the range of flow enthalpies in which carbonaceous deposits can be formed has been identified, obtaining good agreement with the experimental findings. Finally, the deposited substance has been analyzed by means of a set of complementary diagnostic techniques, i.e., SEM, spectroscopy (Raman, FTIR, UV-visible absorption and fluorescence), GC-MS and TGA. It has been found that carbon produced by the interaction of the simulated Titan atmosphere with a solid probe at very high temperatures can be separated into two chemically different fractions, which also include "tholins".
\end{abstract}

Keywords: hypersonic flow; methane; chemical reactions; solid particles; arc-heated facility; Direct Simulation; Monte Carlo method

\section{Introduction}

Titan, the main satellite of Saturn, is the only satellite in the solar system with a dense atmosphere (surface pressure of $1.5 \mathrm{bar}$ ). Below $1000 \mathrm{~km}$, the two major compounds are nitrogen ( 95\%) and methane ( $\sim 2 \%$ in the stratosphere) with a temperature of approximately $90 \mathrm{~K}$. This world has attracted much interest over the years due to its unusual chemistry, which is thought to contain all the necessary ingredients for the emergence of life. For this reason, it has been observed by remote sensing for many years, both from interplanetary probes (Pioneer and Voyager's flybys) and from the Earth. The Voyager 1 (1980), Voyager 2 (1981), Cassini (2004-2017) and Huygens (2005) spacecraft have uncovered physicochemical processes unique in the solar system occurring both in the atmosphere and on the surface [1]. 
The atmosphere and its complex chemical reactions may be regarded as a not-yet-solved problem. Its interaction with solar UV photons and cosmic energetic particles initiates chemical reactions that yield gaseous hydrocarbons and nitriles and, through polymerization processes, solid aerosol particles which grow by coagulation and settle down to the ground [2]. This process results in the production of solid aerosols responsible for the orange haze surrounding Titan. These substances (known as tholins) are the most complex extraterrestrial organic material detected in the solar system [3].

Though the atmosphere of Titan and its ability to produce aerosols in nominal conditions (i.e., at its average temperature of $90 \mathrm{~K}$ ) has extensively been investigated due to the important implications about astrobiology and the "emergence of life"; however, the ability of its atmosphere to produce solid phases at the very high temperatures which are attained during the re-entry of a spacecraft has not been investigated to a comparable extent.

The atmospheric entry of a spacecraft is a critical phase for the success of a planet exploration, and an efficient thermal protection system (TPS) is required to protect the probe against the energy surface flux [4]. The existing studies on this subject have essentially concentrated on the gas dissociation processes which occur when a probe enters the atmosphere of Titan at a very high temperature [5]. These studies are required to properly predict the temperature at the probe surface (in order to design relevant protection systems).

Surprisingly, few studies have been conducted on the formation of solid particles in such processes. To the best of our knowledge, only Vacher et al. [6] have addressed this subject. These authors found solid carbon deposited on the surfaces of an inductively coupled plasma torch operating with a Titan-like atmosphere plasma gas. In the present work, further evidence for the existence of this phenomenon is provided on the basis of a different technique, i.e., a wind tunnel driven by an industrial arc-heated facility operating with nitrogen as working gas (the methane necessary to simulate Titan's atmosphere composition has been added in a plenum-mixing chamber located upstream a supersonic nozzle, [5,7]). Conditions for the formation of such particles are also studied by means of numerical simulation.

This hitherto not yet thoroughly explored phenomenon is of great interest due to its important implications with regard to the aforementioned protection systems (high-speed solid particles may represent a significant threat for any spacecraft or probe).

Apart from these rather technical reasons, the present study has also been motivated by purely academic interests, i.e., by the intention to characterize the mechanisms potentially responsible for the formation and transport of a solid phase in a high-speed flow. Though a vast literature exists about the transport of particles in many fields of fluid-dynamics, the majority of these studies have been conducted for flows which are incompressible and extremely viscous (relevant examples being thermal convection transporting inert ashes inside chimneys and industrial stacks [8], the processing of suspensions, emulsions [9,10] and protein crystals [11], etc.). Only a few studies deal with particles interacting with compressible flow, see e.g., [12-14], or shock waves, see e.g., [15], and most of them have considered inert particles of fixed mass, i.e., non-reacting conditions. By targeting a completely different regime and more complex phenomena, the present work tries to fill a gap still affecting the existing literature.

The present study is articulated as follows: Section 2 contains a description of the hardware and related experimental techniques; at the beginning of Section 3.1, we provide relevant information on the experimentally determined presence of carbon on the surface of the probes used for the tests; moreover, the formation of this substance is investigated in the framework of sophisticated multispecies numerical models considering the effective conditions established inside the SPES (Section 3.1.3); in Section 3.1.4, the deposited substance is analyzed by means of a variegated set of complementary experimental techniques (microscopic, spectroscopic and mass spectrometric methods). It is shown that such carbon (which also features the presence of nanostructured particles) can display two different (more or less dense) morphologies. Section 3.2 is finally devoted to the numerical characterization of the re-entry of the Cassini probe. 


\section{The Facility and (Related)Experimental Techniques}

SPES (Small Planetary Entry Simulator) facility (see Figure 1) belongs to the general category of continuous, open circuit, electric arc-driven, wind tunnels. Its main components are:

- An electric arc-heater (industrial plasma torch, Sulzer-Metco type 9-M), operating with pure inert gases (nitrogen in the present case);

- A mixing chamber where the nitrogen plasma is mixed with cold gases (methane in the present case) to simulate planetary atmospheres;

- Four different conical nozzles (area ratios 4, 20,56, 100) for operations in supersonic and hypersonic flow regime;

- A cylindrical vacuum test chamber (ultimate pressure is in the order of $50 \mathrm{~Pa}$ ).
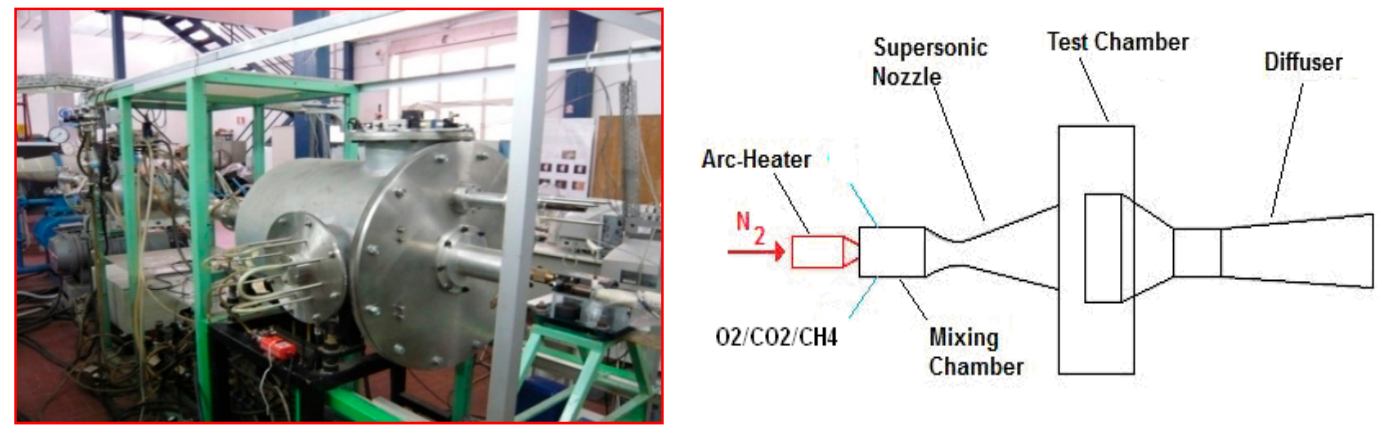

Figure 1. Small Planetary Entry Simulator (SPES) facility (left) and related sketch (right).

Typical SPES operative conditions for entry simulation applications are (order of magnitude): a total mass flow rate of $1 \mathrm{~g} / \mathrm{s}$, an average total enthalpy of $15 \mathrm{MJ} / \mathrm{kg}$ and a total pressure of $5000 \mathrm{~Pa}$.

For the present experiments, the mass-averaged (or bulk) gas enthalpy has been determined at three different positions along the SPES (Figure 1) by the so-called energy balance method [16]. In particular, the measured enthalpies have been calculated as average values resulting from different energy contributors at each position, as illustrated in the following:

(i) Enthalpy of the gas leaving the arc-heater and entering the mixer:

$$
H_{a h}=\frac{P-\left(m_{\mathrm{H}_{2} \mathrm{O}} C \Delta T\right)_{\mathrm{ah}}}{m_{g, a h}}
$$

(evaluated by subtracting the losses due to the cooling water to the input electric power and assuming $\mathrm{C}=4186 \mathrm{~J} / \mathrm{kg})$.

(ii) Enthalpy of the gas leaving the mixer and entering the nozzle:

$$
H_{m c}=\frac{H_{a h} m_{a h}-\left(m_{\mathrm{H}_{2} \mathrm{O}} C \Delta T\right)_{\mathrm{mc}}}{m_{g, t}}
$$

(iii) Enthalpy of the flow at the nozzle exit:

$$
H_{n e}=H_{m c}-\frac{\left(m_{\mathrm{H}_{2} \mathrm{O}} C \Delta T\right)_{n e}}{m_{g, t}}
$$

where the symbol $H$ indicates the total enthalpy $(\mathrm{J} / \mathrm{kg}), P$ is the electrical power $(\mathrm{W}), m$ is the mass flow rate $(\mathrm{kg} / \mathrm{s}), C$ is the water specific heat, $\Delta T(\mathrm{~K})$ is the water temperature jump and the subscripts have the following meaning: ah-arc-heater, g-gas, mc-mixing chamber, ne-nozzle exit, $\mathrm{t}$-total condition (beginning of expansion), $\mathrm{H}_{2} \mathrm{O}$-water, ts-test section. 
During each test the following pressures have been measured $(\mathrm{Pa})$ by electronic vacuum transducers:

- $\quad p_{t}$ at the mixing chamber exit;

- $\quad p_{n e}$ at the nozzle exit;

- $\mathrm{p}_{\mathrm{ts}}$ at the test section;

- $\mathrm{p}_{02}$ impact pressure at the stream centerline.

The uncertainty on the measured total enthalpy evaluation was in the range between $\pm 10 \%$ and $\pm 20 \%$ depending on arc power level, while the uncertainty on the measured pressures was between $\pm 2 \%$ and $\pm 5 \%$.

\section{Results}

\subsection{Ground Model}

\subsubsection{Carbon Formation in SPES (DC Plasma Torch)}

A number of tests have been performed to simulate entry in Titan atmosphere, as for Table 1:

Table 1. Conditions corresponding to the on-the-ground tests.

\begin{tabular}{cccc}
\hline $\mathbf{m}_{\text {gas_N2 }}[\mathrm{g} / \mathbf{s}]$ & $\mathbf{m}_{\text {gas_CH4 }}[\mathrm{g} / \mathbf{s}]$ & $\mathbf{H}_{\text {ne }}[\mathrm{MJ} / \mathbf{k g}]$ & $\mathbf{P}_{\mathbf{0 2}}[\mathrm{Pa}]$ \\
\hline 0.4 & 0.1 & $9.3-24.4$ & $3800-6500$ \\
\hline
\end{tabular}

The same axisymmetric, conical nozzle with two different divergent sections (area ratios 4 and 20) has been used for operations in supersonic and hypersonic flow regime;

The formation of solid carbonaceous deposits occurred on three locations during the SPES tests:

(a) On the water-cooled nozzle walls, both in the convergent and in the divergent sections, see Figure 2a; in this circumstance, the carbonaceous matter appears as a brown substance tenaciously attached to the walls;

(b) On heat flux probes (not cooled), made of brass, Figure 2b; in this case, the particulate appears in the form of black soot;

(c) On a total enthalpy probe (water-cooled), made of steel, Figure 2c; in this circumstance, the carbonaceous matter appears as a brown substance along the probe body, and as black soot in the probe stagnation tip.

Figure $2 b, c$ are extremely interesting as they definitely prove that deposits were formed along the body of (cooled) calorimetric probes and also on the heat flux probes, which were not cooled at all. This apparently innocuous observation has important implications if one considers that it can be used to provide important insights into the underlying mechanisms and the applicability of such results to effective flight conditions (see Section 4 for additional relevant discussions).

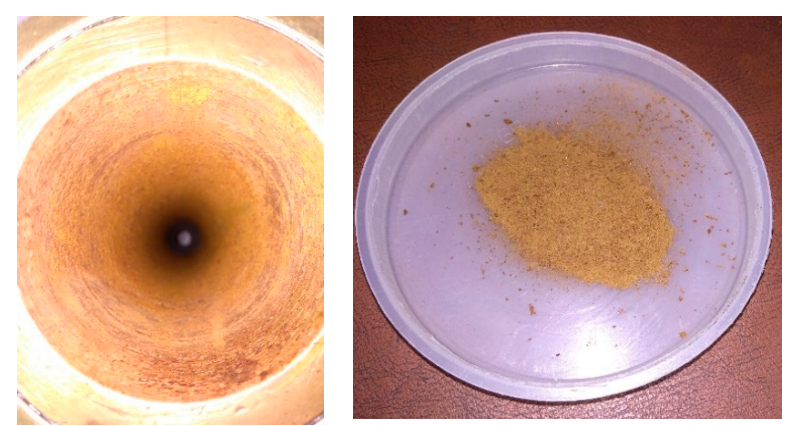

(a) Carbonaceous matter on nozzle walls (left picture) and after being detached from the wall (brown powder shown in the right picture).

Figure 2. Cont. 


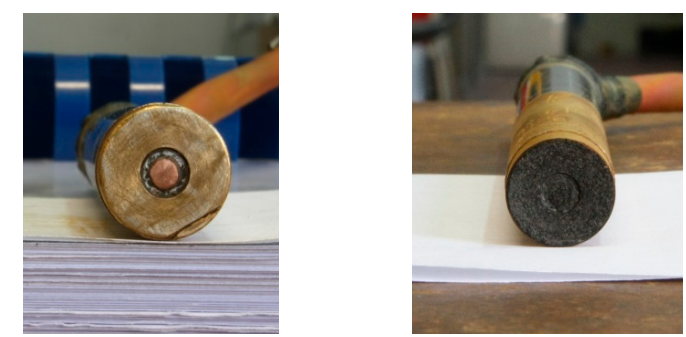

(b) Carbonaceous matter on (not cooled) heat flux probes (black soot).
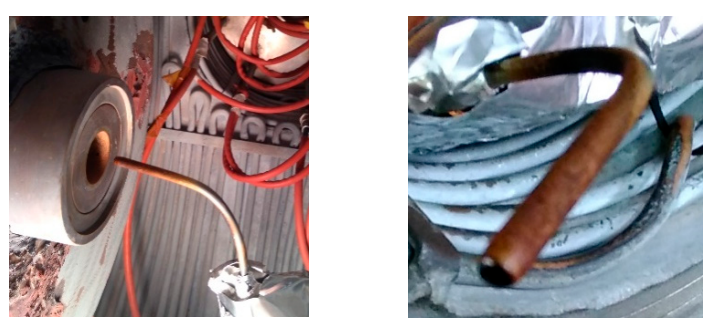

(c) Carbonaceous matter on (water-cooled) total enthalpy probe (brown powder and black soot).

Figure 2. Experimental evidence of carbon deposition on (a) nozzle walls, (b) heat flux probes and (c) total enthalpy probe.

\subsubsection{Carbon Formation in an Induction Plasma Torch}

In this brief section, for the sake of comparison, we wish to recall that Vacher at al. [6] used an inductively coupled plasma torch in their experimental studies of the Titan atmosphere. As shown in Figure 3, carbon powder was found on the ceramic injector and on the quartz tube, whereas no deposition was detected on the part of the tube located inside the induction coil (where plasma temperature is the highest). The carbon powder revealed a high degree of disorder (essentially indicating its amorphous character).

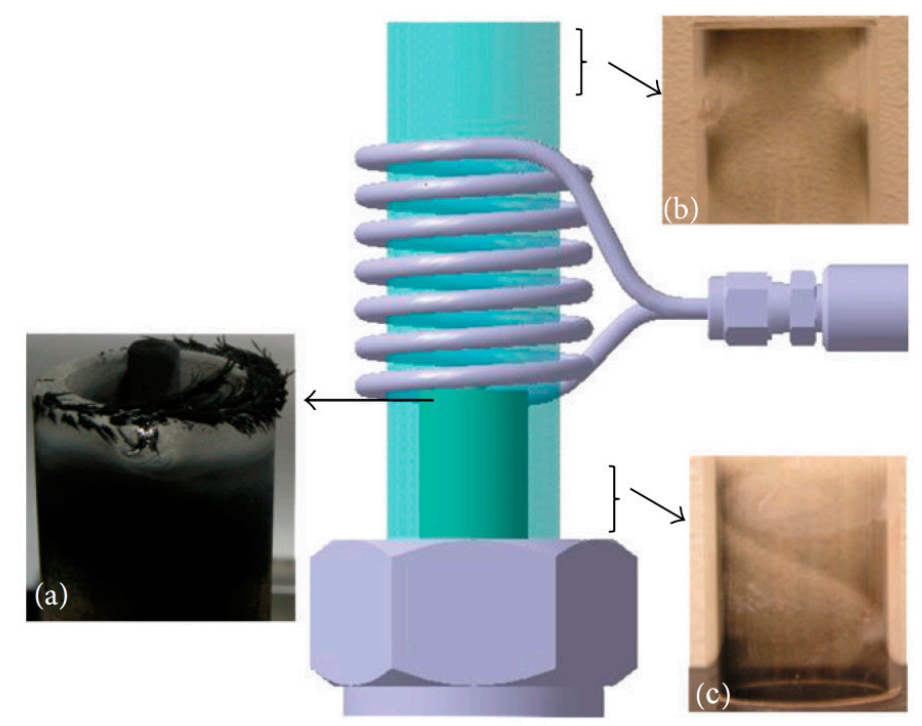

Figure 3. Carbon deposition in Vacher et al.'s torch: Powders location on the ceramic protection (a) and on quartz tube (b) and (c). After Vacher et al., [6] (p. 3). Reproduced under the Creative Commons Attribution License.

Interestingly, even though different particle dimensions were found as a result of different conditions, in no circumstances the observed samples [6] contained nitrogen (indicating the absence of tholins), regardless of their morphology. 


\subsubsection{Carbon Formation-Ground Simulation}

As anticipated at the end of Section 2, the present experimental studies have been supported by means of "ad hoc" numerical simulations. In particular, two different sets of simulations have been conducted in order to assess separately the situation relating to the on-the-ground (SPES-based) tests (see Table 1) and the effective flight conditions relating to the Cassini-Huygens capsule (discussed separately in Section 3.2).

Using the open software CEA from NASA [17], an extended version of the well-assessed Frozen Sonic Flow Method [5] and the experimental test data (mass-averaged enthalpies and pressures), an algorithm has been implemented to calculate gas thermofluid-dynamic gas properties and chemical composition at the arc-heater outlet, mixing chamber inlet (just after mixing of cold methane and hot nitrogen plasma) and finally at the nozzle outlet. The main result from this analysis is that solid carbon originates in the mixing chamber, for total enthalpy values between 10 and $15 \mathrm{MJ} / \mathrm{kg}$.

\subsubsection{Carbon Experimental Characterization}

An array of characterization techniques including: scanning electron microscopy (SEM), spectroscopic techniques (Raman, FTIR, and UV-visible absorption and fluorescence), gas chromatography-mass spectrometry (GC-MS) and Thermogravimetric analysis (TGA), has been applied to the solid deposits described in Section 3.1.1, confirming its carbonaceous nature.

More specifically, the black deposit on the surface of the measuring probe (Figure $2 b, c$ ) was mechanically removed and preliminarily analyzed by Raman spectroscopy and SEM. This material, before solvent extraction, in the following is referred to as whole particulate. More information on the Raman technique can be found in [18].

The brown deposit, also present on the probes, was removed by dipping the probes in dichloromethane (DCM) and sonicating them in an ultrasonic bath for half an hour. The "whole particulate" was added to the sonicated DCM solution and all was then filtered with a Teflon filter (with a porosity of $500 \mathrm{~nm}$ ). All the material was thus separated into two fractions: one soluble in DCM (named DCM-soluble particulate) and one insoluble (DCM-insoluble particulate).

The present analysis has revealed that the brown color of the carbon deposit is due to the rather amorphous-organic (not well graphitized) character of the deposited carbon. This is a result of the decomposition of methane for the experimental conditions considered in this study.

Indeed, the Raman spectrum related to the whole particulate (reported in Figure 4) has shown the characteristic spectral lines of amorphous carbonaceous materials. These are commonly referred to as D-band (peak of disorder) and G-band (peak of graphite). The latter, in fact, is clearly visible in the Raman spectrum of graphite, whose Raman spectrum is also reported in Figure 4 for comparison. The presence of a peak of disorder in the carbon deposit, in addition to the broad bandwidth, is indicative of a carbon material with a high degree of disorder. Most importantly, it is well recognized that the relative intensity of the $\mathrm{D}$ and $\mathrm{G}$ peaks, $I(\mathrm{D}) / I(\mathrm{G})$ can be related to the size of graphitic domains [19]. From the measured Raman spectra, we obtained an average value of $I(\mathrm{D}) / I(\mathrm{G})$ of approximately 0.85 , which corresponds to a value of $L_{\mathrm{a}}$ slightly lower than $1 \mathrm{~nm}$. A background in the Raman spectrum of the carbon deposit was also measured. This feature, commonly attributed to photoluminescence/fluorescence phenomena, can be ascribed to the presence of an organic matrix (organic carbon).

Interestingly, the Raman spectrum of the carbon deposit was very similar to those characteristic of carbonaceous particulates produced by hydrocarbon fuel combustion [19]; therefore, it is reasonable to speculate that these deposits have a carbonaceous structure similar to soot. 


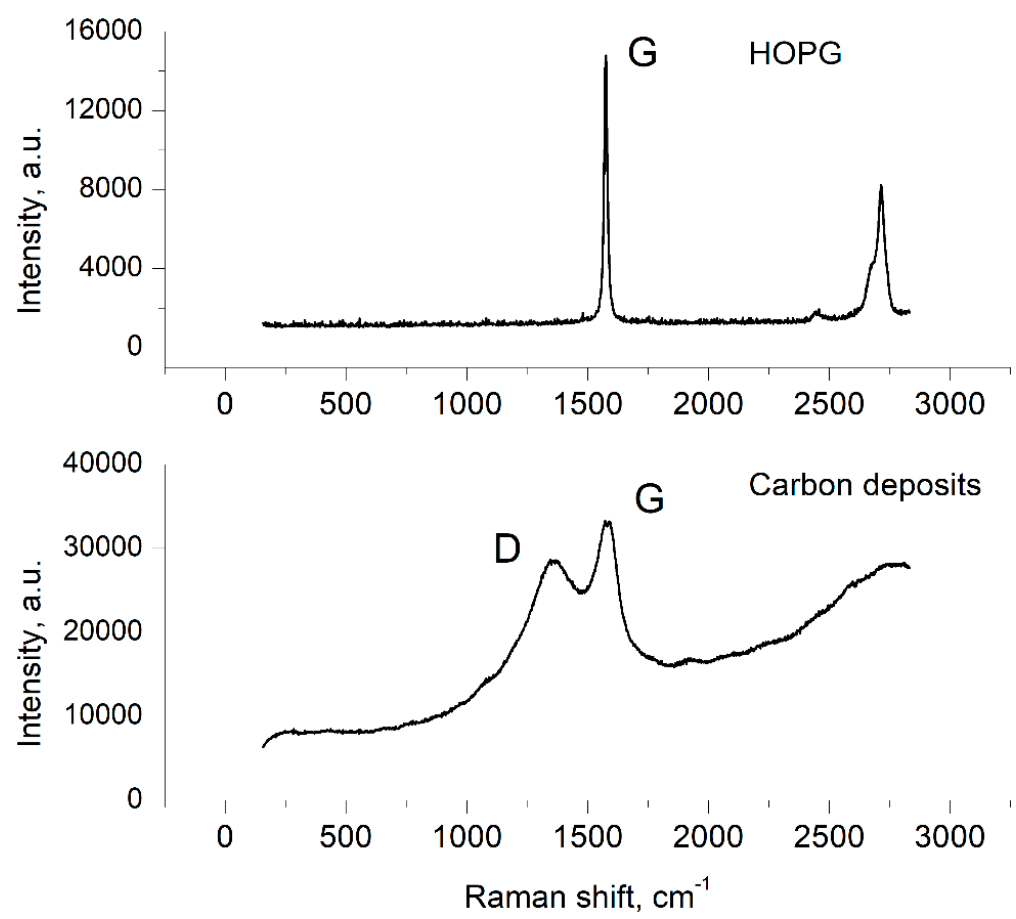

Figure 4. Upper panel: Raman spectrum of highly oriented pyrolytic graphite (HOPG). Lower panel: Raman spectrum of whole particulate.

Figure 5 (and its inset) show the results provided by SEM with EDX (energy-dispersive X-ray spectroscopy) analysis applied to the whole particulate before solvent extraction. SEM analysis was not successful in providing information on the morphology of the samples, mainly composed of heterogeneous blocks. Nevertheless, the EDX-based approach was instrumental in making evident the dominant presence of carbon, with traces of metals (probably released from the arc electrodes).

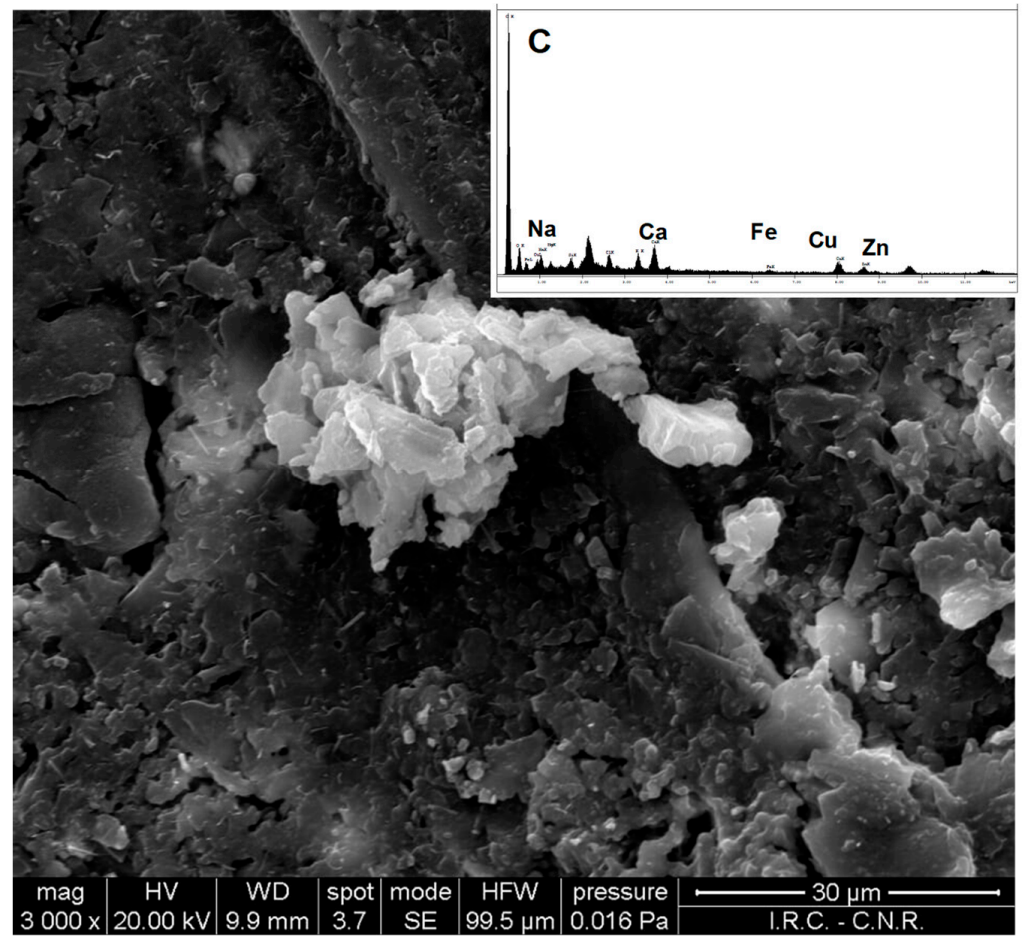

Figure 5. SEM image of whole particulate. The EDX (energy-dispersive X-ray spectroscopy) spectrum is reported in the inset. 
The DCM-soluble particulate was analyzed by GC-MS and UV-visible spectroscopy (absorption and fluorescence). More details on the techniques are reported in [20]. The GC-MS revealed the presence of very a low amount of small aromatic molecules with oxygenated and nitrogenous functionalities and the total absence of polycyclic aromatic hydrocarbons (PAH), typically formed in combustion and pyrolysis processes from hydrocarbon fuels [21]. The absorption and fluorescence spectra of DCM-soluble particulate, reported in Figures 6 and 7, respectively, present features that can typically be ascribed to tholins, the aforementioned very complex substances consisting of reddish-brown polymeric material with aromatic moieties and oxygen and nitrogen heteroatoms [22,23].

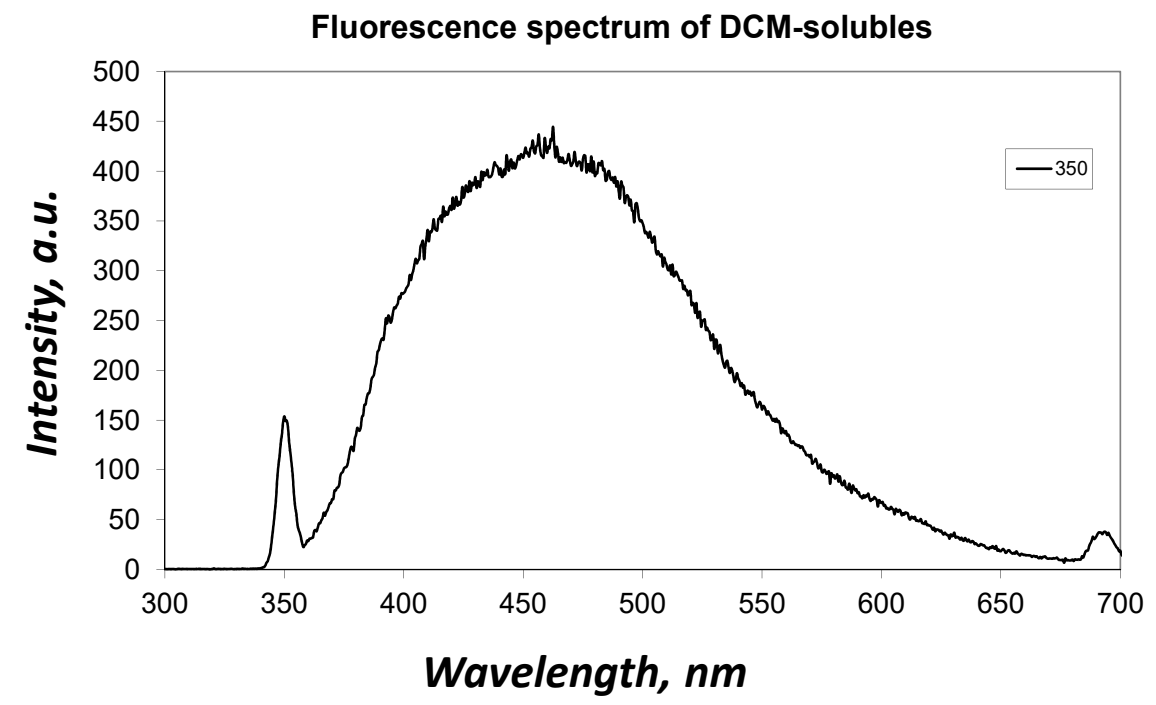

Figure 6. Fluorescence emission spectrum $(\lambda \operatorname{exc}=350 \mathrm{~nm})$ of the DCM soluble particulate.

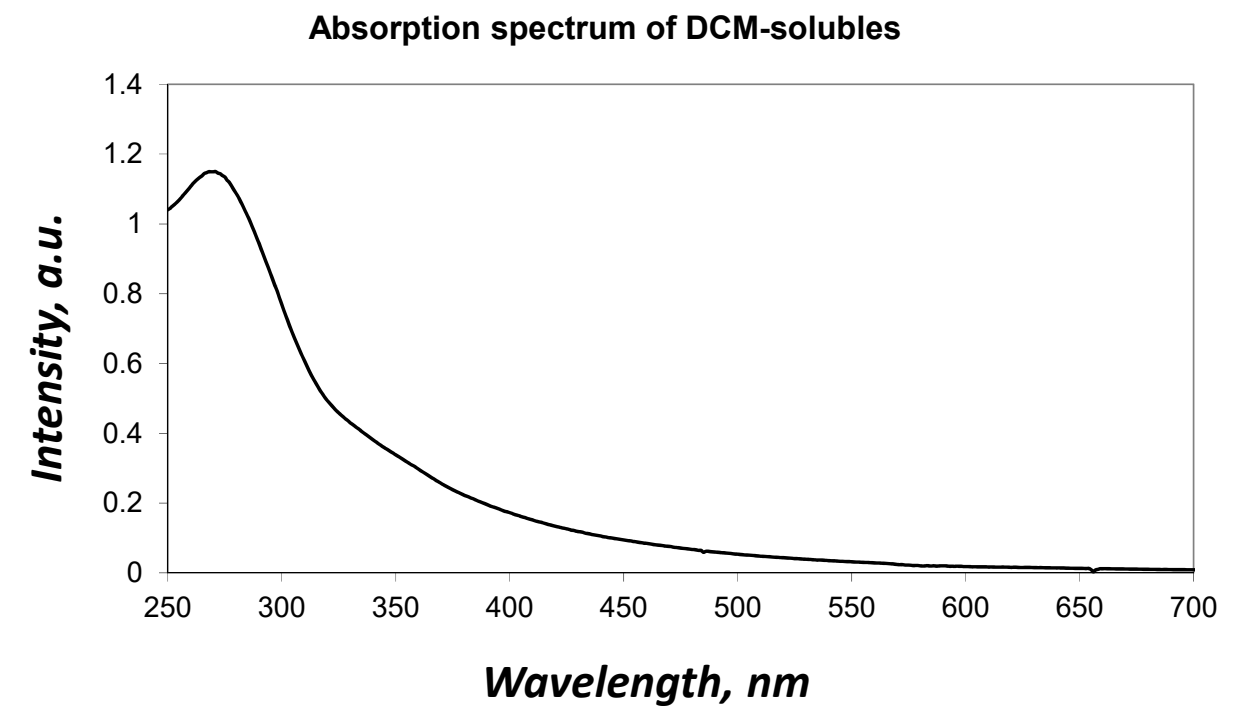

Figure 7. UV-visible absorption spectrum of the DCM-soluble particulate.

Vacher at al. [6] found carbonaceous samples deposited on the probes with amorphous character but without nitrogen, indicating the absence of tholins. Anyway, those authors analyzed only the whole particulate, without solvent extraction, and without the implementation of techniques able directly detecting nitrogen functionalities. In the present work, the solvent extraction allowed isolating the tholins fraction from the amorphous carbonaceous core, enabling its detection.

FTIR spectroscopy, applied to the DCM-insoluble particulate, has provided evidence for the essentially carbonaceous nature of the substance though with high amorphous character, due to a large 
presence of aliphatic functionalities. The presence of oxygenated functionalities and the absence of nitrogenous functionalities are remarkable. The TGA profile in air of the DCM-insoluble particulate presented two mass losses, indicating the heterogeneity of the sample. The main mass loss occurred at a temperature typical of carbonaceous particulate (soot) oxidation (around $600{ }^{\circ} \mathrm{C}$ ), whereas the second one occurred at a lower temperature (around $350{ }^{\circ} \mathrm{C}$ ).

The brown powder deposited on nozzle walls (Figure 2a) was found to be soluble in DCM and very similar to the DCM-soluble particulate from the probes, therefore only the analyses on brown deposits from probes have been reported here.

\subsection{Carbon Formation-Flight Simulation}

Titan's atmosphere is basically made up of three chemical species: nitrogen $\left(\mathrm{N}_{2}\right)$, methane $\left(\mathrm{CH}_{4}\right)$ and argon (AR) whose composition is almost constant with altitude. The molar fractions are about: $\mathrm{X}_{\mathrm{N} 2}=0.95, \mathrm{X}_{\mathrm{CH} 4}=0.03, \mathrm{X}_{\mathrm{AR}}=0.02$. Due to the high number of reactions produced by high energy during a capsule entry path, in the present work, the atmosphere has been considered made of 18 chemical species: $\mathrm{N}_{2}, \mathrm{CH}_{4}, \mathrm{CH}_{3}, \mathrm{CH}_{2}, \mathrm{CH}, \mathrm{C}_{2}, \mathrm{H}_{2}, \mathrm{CN}, \mathrm{NH}, \mathrm{HCN}, \mathrm{N}, \mathrm{C}, \mathrm{H}, \mathrm{AR}, \mathrm{N}_{2}^{+}, \mathrm{CN}^{+}, \mathrm{N}^{+}, \mathrm{C}^{+}, \mathrm{AR}$. The chemical model by Savajano et al. [24] has been used. This model is made up of 221 reactions: 205 dissociations, 11 exchanges, 5 ionizations. The numerical study has been carried out in the altitude interval from 180 to $470 \mathrm{~km}$. The Huygens-Cassini capsule, depicted schematically in Figure 8, was a $60^{\circ}$ half-angle sphere-cone with a diameter (D) of $2.7 \mathrm{~m}$.

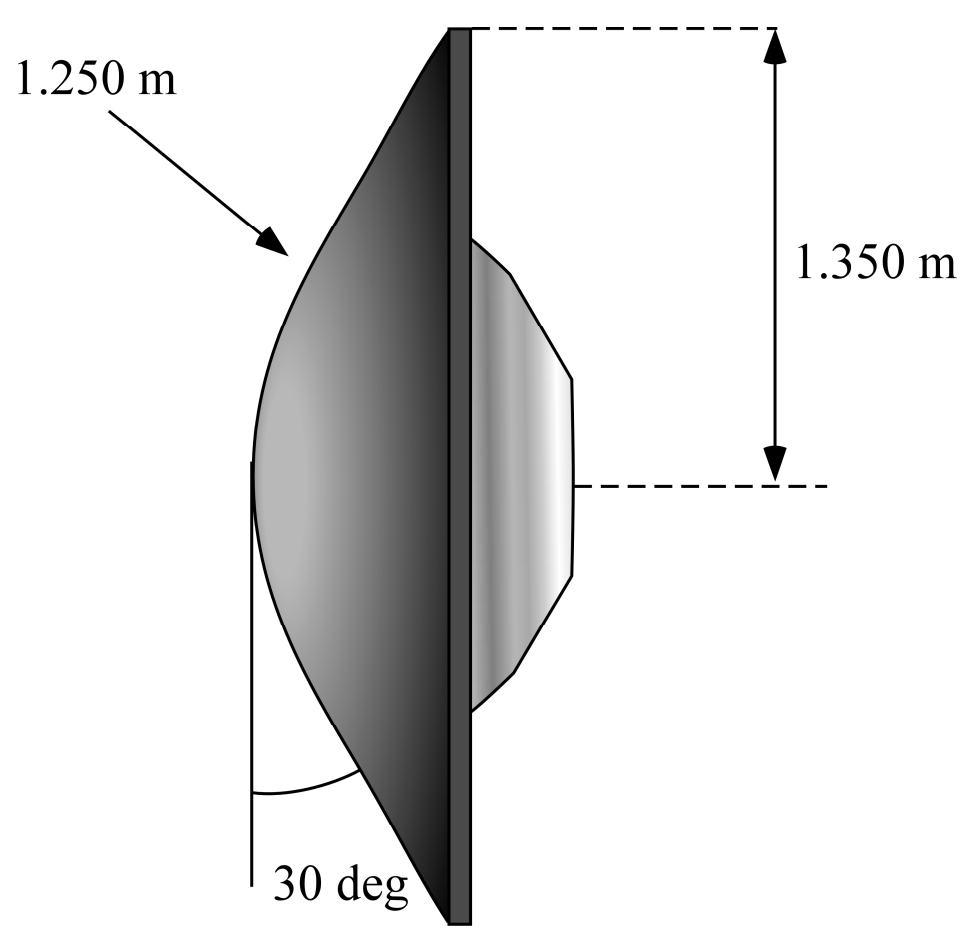

Figure 8. Schematic of Cassini-Huygens capsule.

Data about the Cassini-Huygens entry are available in the study by Wright et al. [25], who computed the related trajectory; Figure 9a,b verifies that the flow field around the capsule in Titan entry is in the transitional (continuum low density) regime. In fact, according to:

(i) Vallerani [26], the transitional regime for a blunt body is defined in terms of the Reynolds number downstream a normal shock wave $\operatorname{Re}_{2 \mathrm{D}}$ by $10^{-1}<\operatorname{Re}_{2 D}<10^{4}\left(\operatorname{Re}_{2 \mathrm{D}}=\rho_{\infty} \mathrm{V}_{\infty} \mathrm{D} / \mu_{2}\right.$, being $\mu_{2}$ the viscosity downstream a normal shock wave);

(ii) Moss [27], in terms of the global Knudsen number $\mathrm{Kn}_{\infty \mathrm{D}}$ by $10^{-3}<\mathrm{Kn}_{\infty \mathrm{D}}<50\left(\mathrm{Kn}_{\infty \mathrm{D}}=\lambda_{\infty} / \mathrm{D}\right.$, being $\lambda_{\infty}$ the mean free path). 


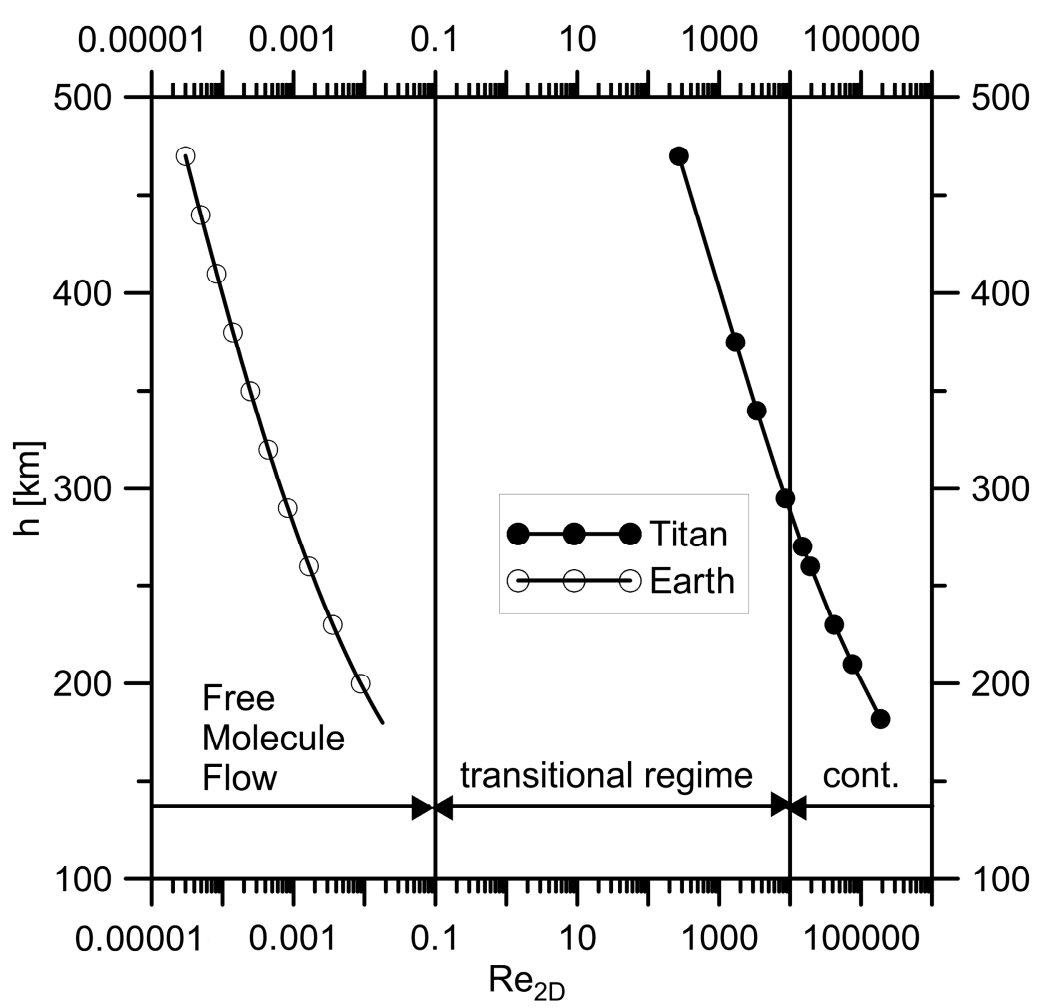

(a)

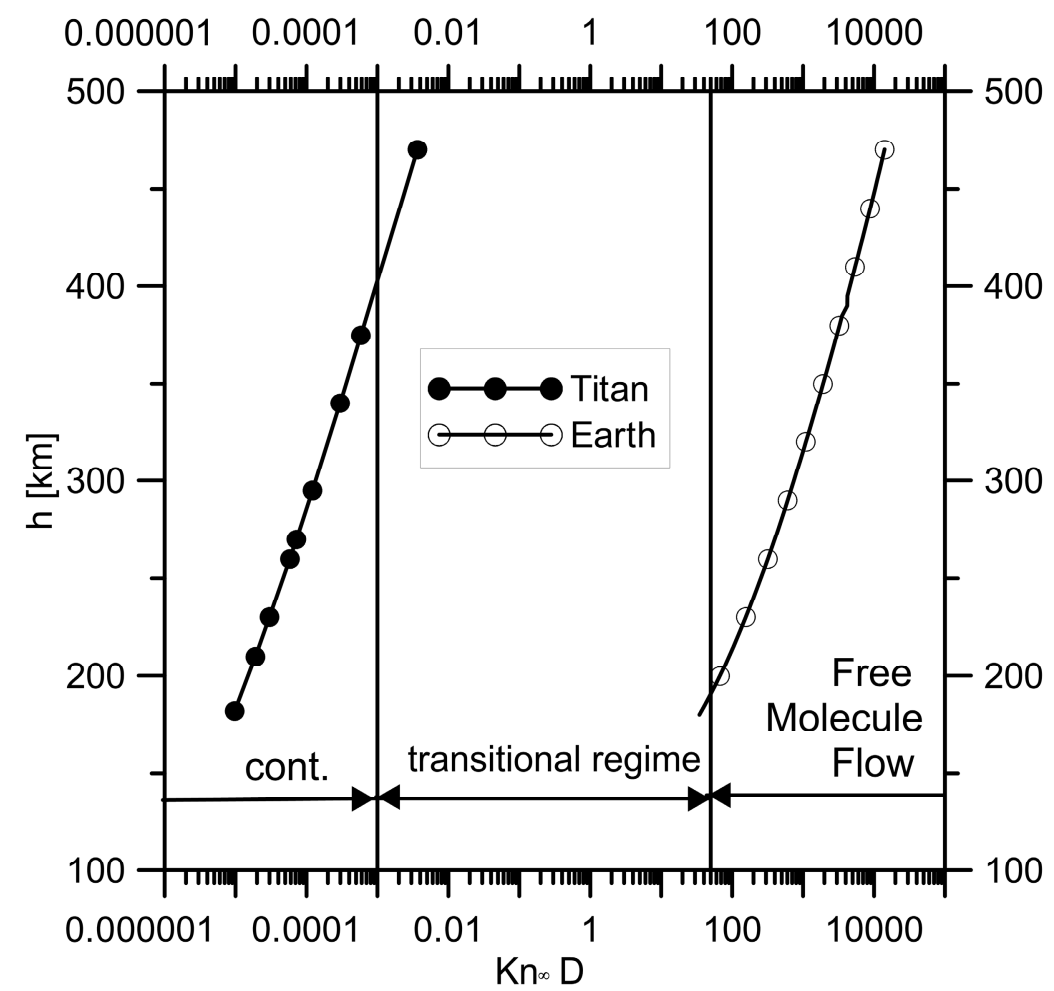

(b)

Figure 9. Profiles of (a) Reynolds number downstream a normal shock wave and (b) Knudsen number as functions of altitude.

As the flow field is in the transitional regime, it can be solved by means of the direct simulation Monte Carlo (DSMC) method [28-30]; more specifically, the DS2V-4.5 64-bit [31] code has been used. This code offers the user the opportunity to simulate in a relatively straightforward way the absorption 
process of molecules impinging onto the surface of a body. Thereby, it is particularly suited for the simulation of the formation process of a carbon layer. In particular, DS2V-4.5 64 bits makes it possible to diversify the type of molecule-surface interaction for each molecule. In the present case, when interacting with the surface, all molecules, except for carbon $(C)$, were considered re-emitted from the surface by a diffusive re-emission process at the wall temperature of $300 \mathrm{~K}$ (while carbon molecules were considered absorbed by the surface, therefore, eliminated from the calculation).

Before embarking into the description of the results obtained with this model, we would like to point out that it should be regarded as a reliable representation of reality only for relatively small temperatures of the wall such as that indicated above. As reported by Bouilly [32], the effective thermal protection system of the Huygens probe was based on an ablative phenolic resin. More specifically, the shield was made of AQ 60/I, i.e., a reinforced felt of short silica fibers impregnated with a phenolic resin. Due to its non-mineral bonding agent, this material undergoes pyrolysis for temperatures between $470 \mathrm{~K}$ and $1270 \mathrm{~K}$ and, accordingly, "ablation" occurs (which is beyond the capabilities of the model described above). The physical relevance of the results presented in this section should, therefore, be considered limited to the conditions for which they have been determined, i.e., a relatively small wall temperature.

Although these simulations may not capture the underlying physics quantitatively for larger temperatures, they are used here to identify the physical mechanisms responsible for solid carbon accumulation. Indeed, the possibility to treat carbon molecules as entities being absorbed by the surface gives effective insights into the generation of the carbon layer on the capsule surface, whose thickness can be quantified by the flux of carbon atoms (C) impinging onto the surface.

In particular, as shown in Table 2, we have conducted four computer tests. At these altitudes the flow total enthalpy is such that carbon atoms can be produced by dissociation of $\mathrm{CH}_{4}, \mathrm{CH}_{3}, \mathrm{CH}_{2}$, etc., $\eta_{\infty}$ is the number density or the number of particles (atoms and molecules) per unit of volume.

Table 2. DS2V-4.5 64-bit input data and free stream enthalpy.

\begin{tabular}{ccccc}
\hline Test & $\mathbf{h}[\mathbf{k m}]$ & $\mathbf{V}_{\infty}[\mathbf{m} / \mathbf{s}]$ & $\eta_{\infty}\left[\mathbf{1} / \mathbf{m}^{3}\right]$ & $\boldsymbol{H}_{\infty}[\mathbf{M J} / \mathbf{k g}]$ \\
\hline 1 & 470 & 6167 & $1.295 \times 10^{20}$ & 19.2 \\
2 & 375 & 6049 & $8.117 \times 10^{20}$ & 18.5 \\
3 & 340 & 5886 & $1.590 \times 10^{21}$ & 17.5 \\
4 & 295 & 5490 & $3.916 \times 10^{21}$ & 15.3 \\
\hline
\end{tabular}

Wright [25] presented the entry trajectory in terms of the flight time ( $t[\mathrm{~s}])$. For the purpose of the present study, the corresponding altitude was determined by means of the match of the free stream density provided by Wright and that provided by Yelle's model [33]. Temperature, gas composition, etc. used in the present computations, are those from Yelle's model.

The thickness of the carbon layer, as a function of curvilinear abscissa $\sigma[\mathrm{m}]$ from the stagnation point, deposited along the surface of the capsule is expected to be closely linked to the flow of carbon atoms impinging onto the surface. Along these lines, Figure 10a shows the profile of the number flux $\left(\mathrm{N}_{\mathrm{C}}\right)$ of carbon atoms (i.e., the number of carbon atoms impinging on the surface per unit surface and per second $\mathrm{m}^{-2} \mathrm{~s}^{-1}$ ), for example, for Test 1 . The profile looks pretty scattered and, as expected, it assumes the maximum value at the stagnation point $(\sigma=0 \mathrm{~m})$ where the shock wave is stronger compared with that of every point along its front; correspondingly gas gets maximum reactivity. Because of the data scatter, the flux of carbon atoms (C) are compared by means of best fit curves. Computation related to Test 4 is not shown in Figure 10b; $N_{C}$ for Test 4 is negligible. Even though Test 1 is the most energized, however, the formation of carbon atoms is the lowest; the generation of carbon atoms is due both to the energy level and the density of the flow. 


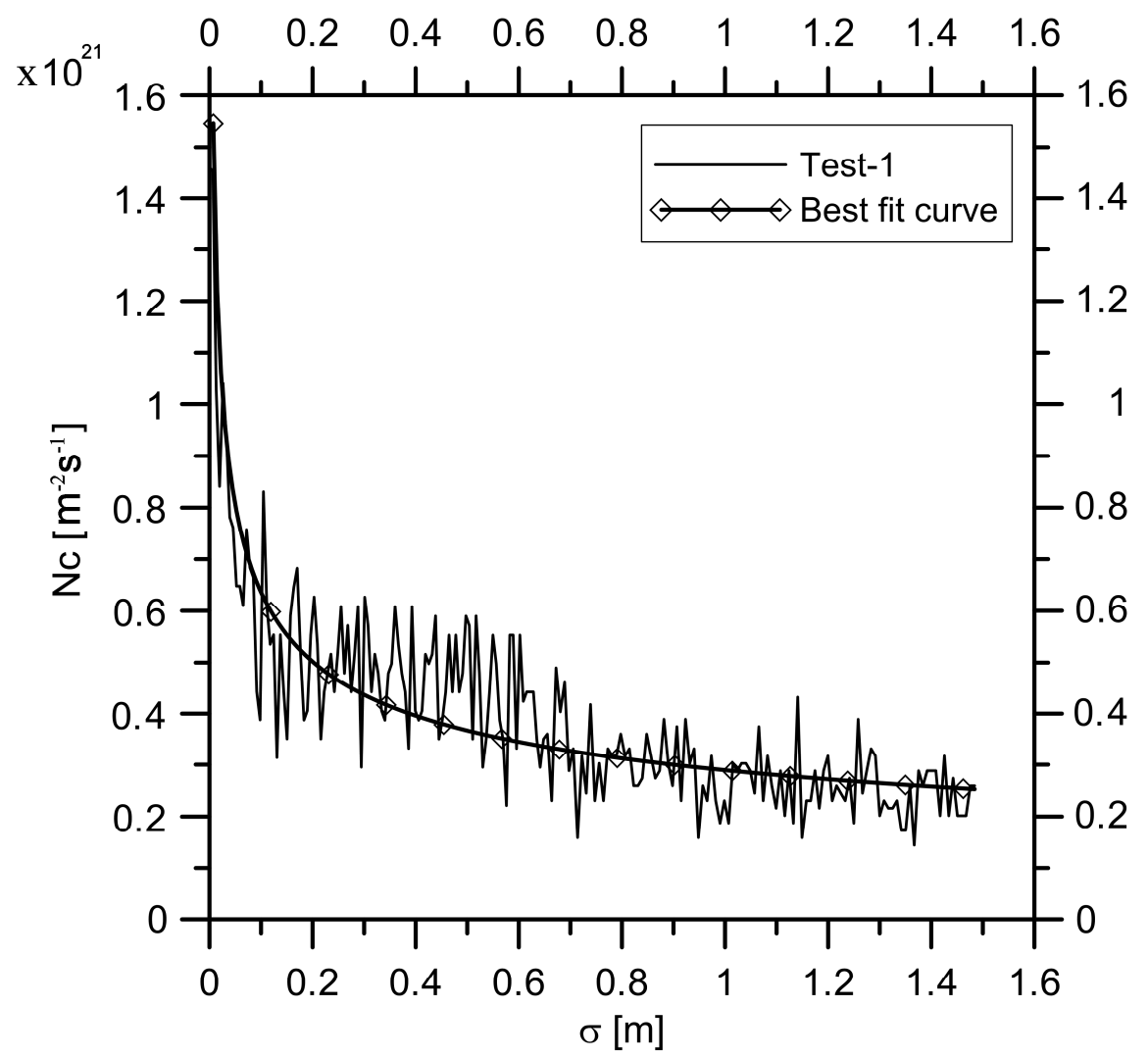

(a)

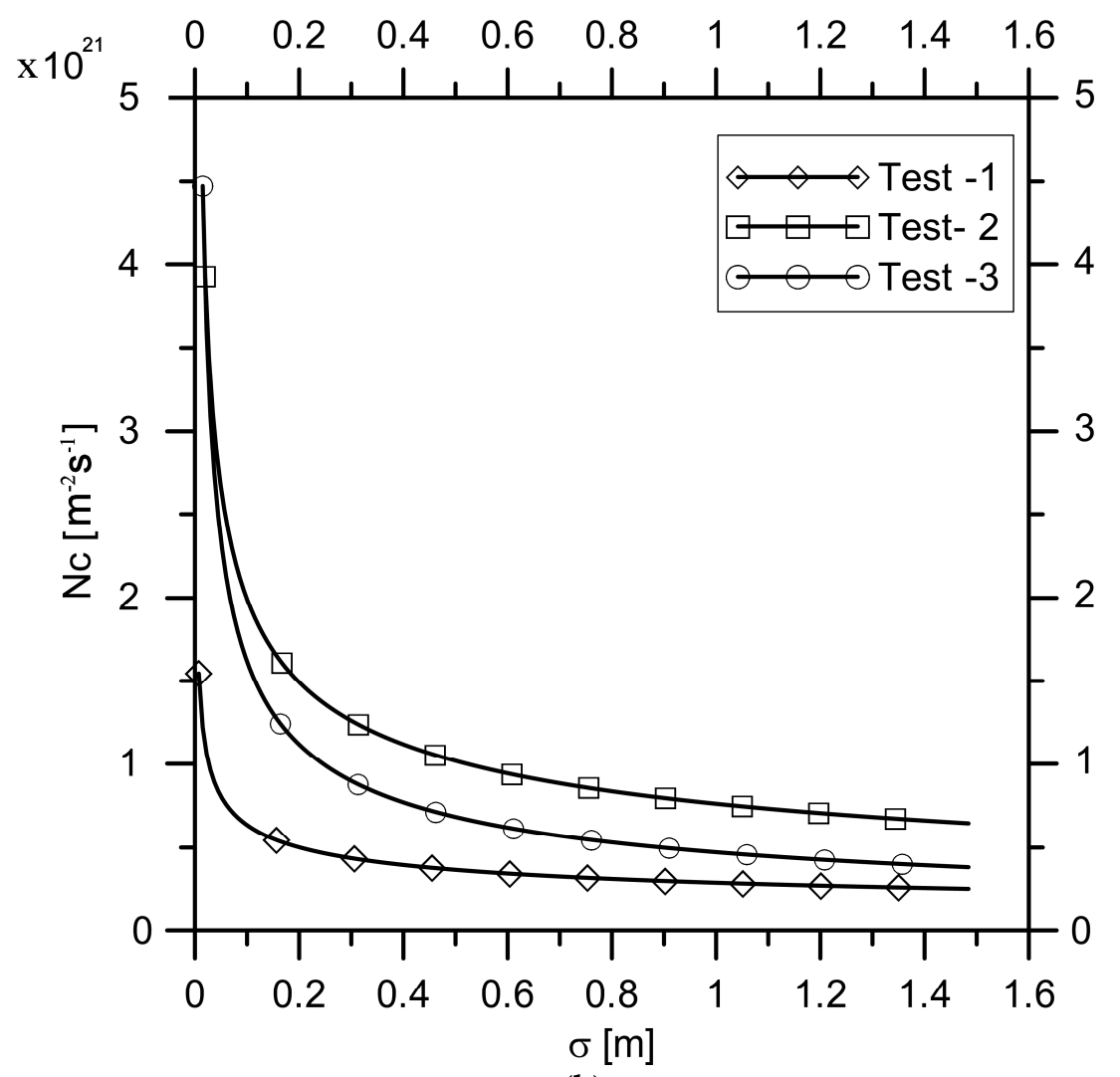

(b)

Figure 10. (a) Profile of Carbon flux to the capsule surface for Test 1 and related best fit curve $(\ln (y)=-0.3421436438 \ln (x)+47.11408227),(b)$ comparison of the best fit curves for three tests. 
On the basis of these tests, the formation of carbon during effective re-entry conditions can be considered significant for enthalpies not exceeding (approximately) $16 \mathrm{MJ} / \mathrm{kg}$.

\section{Discussion}

In all cases considered in the present study, on the basis of the CEA simulations, it can be concluded that all methane was essentially cracked before reaching the SPES nozzle exit (where the static temperature was more or less $1500 \mathrm{~K}$ ). As illustrated in Section 3.1.1, during the tests, the formation of deposits was not limited to the body of the (cooled) calorimetric probes. They were also formed on the heat flux probes, which were not experiencing any cooling. These arguments can, therefore, be seen as an indirect demonstration of the potential relevance of such dynamics to the effective flight conditions that are established when a probe experiences high-temperature re-entry.

It is also worth mentioning that we found the chemical composition of the material along the body of the calorimeter probe to vary depending on the position. While along the probe body (where the effects of the cooling are more significant) the chemical composition was relatively similar to that of the aforementioned tholins (that can also be formed naturally by solar ultraviolet irradiation or cosmic rays in the cold atmosphere of Titan), on the nose (where a shock wave is formed and the temperature is extremely high) the substance was essentially pure carbon. The latter results are essentially in agreement with the independent findings by Vacher et al. [6], who, using a different facility, found solid carbon along hot walls and parts (Section 3.1.2).

These observations, together with the outcomes of the numerical simulations presented in Section 3.1.3 further confirm that solid carbon can primarily be formed as a result of the interaction of the considered gas mixture with a shock wave and the related high temperatures, which lead to complex chemical reactions. It is not a consequence of cooling effects (which rather tend to support the emergence of tholins).

With regard to effective flight conditions of the Cassini-Huygens capsule, on the basis of the numerical simulations reported in Section 3.2, the formation of solid carbon should still be expected for not too high values of the total enthalpy (values similar to those for which it was found using the SPES). Obviously, for such a case it cannot be excluded that tholins may also be formed and accumulated on the heat shield. With regard to real re-entry conditions, it should be highlighted that the thermal shield of a vehicle is generally subjected to a variety of conditions [32]. Its temperature grows with time. This means that, on the basis of the present study, in addition to pure solid carbon also the formation of tholins might be expected at some stages.

We wish to recall that during our tests, as soon as soot was formed, it could dramatically affect temperature measurements. Therefore, soot would likely affect the heat flux, the front shield and the ablator of any hypersonic vehicle.

\section{Conclusions and Future Directions}

Understanding the formation of solid phases in atmospheres containing a significant percentage of methane is a topic of great importance from several points of view. As an example, in the atmosphere of Titan, solid particles can be produced due to natural processes (giving rise to the so-called tholins, which so much attention have attracted over recent years owing to their remarkable implications in the field of astrobiology) or at very high temperatures when a probe or vehicle enters the atmosphere of this planet (as a result of the complex chemical reactions that are enabled in the hypersonic regime). In the latter case, the nucleation of solid particles or the formation of accumulation layers can have potentially dangerous effects, which need to be properly understood and mitigated by designing adequate protection systems.

The resurgence in interest in these phenomena has recently been driven by the new missions that NASA is planning to the Ice Giants. The atmospheres of most of the outer planets of the solar system indeed contain appreciable percentages of methane. This is the case for instance of Neptune, whose atmosphere is made up predominantly of hydrogen and helium, but also includes some methane. 
Uranus also has methane in its atmosphere, and traces of this gas can also be found in the atmospheres of Jupiter and Saturn.

In June 2017, NASA published the Ice Giants Pre-Decadal Survey Mission Study Report, which focused on science priorities and mission concepts for those planets. ESA has recently performed two studies (January and July 2019) which analyzed the feasibility of stand-alone elements (orbiter and probes) provided by ESA as a part of a NASA led mission to the Uranus or Neptune systems. These probes will experience extreme heating rates to the thermal protection system from convective and radiative transport. With a Neptune probe under consideration as a possible European contribution to the Ice Giants mission, the development of predictive tools on the basis of dedicated experimental and theoretical analysis will be crucial for taking the necessary countermeasures to ensure the success of these and other future missions.

The present study was aimed at the characterization of methane-driven phenomena in order to provide mission analysts and aerospace engineers with the necessary knowledge to include related effects in the design of future missions and planetary protection systems.

In addition to practical applications, the present analysis was also motivated by academic curiosity, i.e., by the intention to investigate the spontaneous formation of organic solid phases in a high-enthalpy flow. Most past studies have been limited to the nucleation and growth of crystals of proteins or other macromolecular substances in very viscous liquids or solutions.

We have analyzed the carbonaceous nature of the particulate matter formed as a result of the complex chemical reaction occurring during the re-entry in the Titan atmosphere (hypersonic regime) via various diagnostic techniques such as SEM, Raman, FTIR, UV-visible absorption and fluorescence spectroscopy, GC-MS and TGA. The interpretation of experimental findings has been supported by dedicated numerical simulations based on the NASA CEA code and the Direct Simulation Monte Carlo (DSMC) method. These simulations have confirmed that solid particles can effectively be formed for total enthalpy values between 10 and $15 \mathrm{MJ} / \mathrm{kg}$ and that they are not a result of localized cooling effects.

Future studies shall be devoted to (1) simulate the entire formation process of solid carbonaceous particles in the hypersonic regime by means of a multispecies chemical model fully integrated into a Navier-Stokes-equations-based Computational Fluid-Dynamics (CFD) platform and (2) consider other atmospheres, which also contain significant percentages of methane such as those of Uranus and Neptune.

Author Contributions: Data curation, A.E., G.Z., C.A., B.A., M.C., P.M. and C.R.; Formal analysis, M.L.; Investigation, A.E., G.Z., C.A., B.A., M.C., P.M. and C.R.; Methodology, A.E. and G.Z.; Software, G.Z.; Supervision, A.E. and M.L.; Visualization, A.E.; Writing—original draft, M.L.; Writing—review and editing, M.L. All authors have read and agreed to the published version of the manuscript.

Funding: This research received no external funding.

Conflicts of Interest: The authors declare no conflict of interest.

\section{References}

1. Dubois, D.; Carrasco, N.; Petrucciani, M.; Vettier, L.; Tigrin, S.; Pernot, P. In situ investigation of neutrals involved in the formation of Titan tholins. Icarus 2019, 317, 182-196. [CrossRef]

2. Bernard, J.M.; Colla, P.; Coustenis, A.; Raulina, F. Experimental simulation of Titan's atmosphere: Detection of ammonia and ethylene oxide. Planet. Space Sci. 2003, 51, 1003-1011. [CrossRef]

3. Carrasco, N.; Gautier, T.; Es-sebbar, E.; Pernot, P.; Cernogora, G. Volatile products controlling Titan's tholins production. Icarus 2012, 219, 230-240. [CrossRef]

4. Wang, J.; Han, F.; Lei, L.; Lee, C. Numerical Study of High-Temperature Nonequilibrium Flow around Reentry Vehicle Coupled with Thermal Radiation. Fluid Dyn. Mater. Process. 2020, 16, 601-613. [CrossRef]

5. Esposito, A.; Lappa, M. Experimental and Theoretical Verification of the Frozen Sonic Flow Method for Mixtures of Polyatomic Gases. AIAA J. 2019, 58, 265-277. [CrossRef] 
6. Vacher, D.; Menecier, S.; Dudeck, M.; Dubois, M.; Devouard, B.; Petit, E. Solid Carbon Produced in an Inductively Coupled Plasma Torch with a Titan Like Atmosphere. Int. J. Aerospace Eng. 2013, 2013, 546385. [CrossRef]

7. Carandente, V.; Savino, R.; Esposito, A.; Zuppardi, G.; Caso, V. Experimental and numerical simulation, by an arc-jet facility, of hypersonic flow in Titan's atmosphere. Exp. Therm. Fluid. 2013, 48, 97-101. [CrossRef]

8. Lappa, M. On the transport, segregation and dispersion of heavy and light particles interacting with rising thermal plumes. Phys. Fluids 2018, 30, 033302. [CrossRef]

9. Lappa, M. Stationary Solid Particle Attractors in Standing Waves. Phys. Fluids 2014, 26, 013305. [CrossRef]

10. Lappa, M. Time reversibility and non-deterministic behaviour in oscillatorily sheared suspensions of non-interacting particles at high Reynolds numbers. Comput. Fluids 2019, 184, 78-90. [CrossRef]

11. Lappa, M.; Piccolo, C.; Carotenuto, L. Numerical and experimental analysis of periodic patterns and sedimentation of lysozyme. J. Cryst. Growth 2003, 254, 469-486. [CrossRef]

12. Lappa, M.; Esposito, A.; Aponte, F.; Allouis, C. High Velocity Infrared Thermography and Numerical Trajectories of Solid Particles in Compressible Gas Flow. Powder Technol. 2019, 343, 671-682. [CrossRef]

13. Jewell, J.S.; Parziale, N.J.; Leyva, I.A.; Shepherd, J.E. Effects of shock-tube cleanliness on hypersonic boundary layer transition at high enthalpy. AIAA J. 2017, 55, 332-338. [CrossRef]

14. Chuvakhov, P.V.; Fedorov, A.V.; Obraz, A.O. Numerical modelling of supersonic boundary-layer receptivity to solid particulates. J. Fluid Mech. 2019, 859, 949-971. [CrossRef]

15. Lappa, M.; Drikakis, D.; Kokkinakis, I. On the propagation and multiple reflections of a blast wave travelling through a dusty gas in a closed box. Phys. Fluids 2017, 29, 033301. [CrossRef]

16. Pope, R.B. Measurements of enthalpy in low-density arc-heated flows. AIAA J. 1968, 6, 103-110. [CrossRef]

17. Gordon, S.; McBride, B.J. Computer Program for Calculation of Complex Chemical Equilibrium Compositions and Applications I. Analysis. NASA RP-13111994. Available online: https://ntrs.nasa.gov/archive/nasa/casi. ntrs.nasa.gov/19950013764.pdf (accessed on 10 June 2020).

18. Commodo, M.; Joo, P.H.; De Falco, G.; D’Anna, A.; Gülder, O.L. Raman Spectroscopy of Soot Sampled in High-Pressure Diffusion Flames. Energy Fuels 2017, 31, 10158-10164. [CrossRef]

19. Ferrari, C.; Robertson, J. Raman spectroscopy of amorphous, nanostructured, diamond-like carbon, and nanodiamond. Philos. Trans. R. Soc. Lond. A 2004, 362, 2477-2512. [CrossRef]

20. Gargiulo, V.; Apicella, B.; Russo, C.; Stanzione, F.; Tregrossi, A.; Millan, M.; Ciajolo, A. Structural Characterization of Large Polycyclic Aromatic Hydrocarbons. Part 2: Solvent-Separated Fractions of Coal Tar Pitch and Naphthalene-Derived Pitch. Energy Fuels 2016, 30, 2574-2583. [CrossRef]

21. Panariello, M.; Apicella, B.; Armenante, M.; Bruno, A.; Ciajolo, A.; Spinelli, N. Analysis of polycyclic aromatic hydrocarbon sequences in a premixed laminar flame by on-line time-of-flight mass spectrometry. Rapid Commun. Mass Spectrom. 2008, 22, 573-581. [CrossRef]

22. Hodyss, R.; McDonald, G.; Sarker, N.; Smith, M.A.; Beauchamp, P.M.; Beauchamp, J.L. Fluorescence spectra of Titan tholins: In-situ detection of astrobiologically interesting areas on Titan's surface. Icarus 2004, 171, 525-530. [CrossRef]

23. Carrasco, N.; Schmitz-Afonso, I.; Bonnet, J.-Y.; Quirico, E.; Thissen, R.; Dutuit, O.; Bagag, A.; Laprévote, O.; Buch, A.; Giulani, A.; et al. Chemical Characterization of Titan's Tholins: Solubility, Morphology and Molecular Structure Revisited. J. Phys. Chem. A 2009, 113, 11195-11203. [CrossRef] [PubMed]

24. Savajano, R.; Sobbia, R.; Gaffuri, M.; Leyland, P. Reduced Chemical Kinetic Model for Titan Entries. Int. J. Chem. Eng. 2011, 2011, 970247. [CrossRef]

25. Wright, J.; Hollis, B.R.; Bose, D.; Walpot, L. Post-Flight Aerothermal Analysis of Huygens Problem. In Proceedings of the 3rd International Workshop Planetary Probe, Anavyssos, Attiki, Greece, 27 June-1 July 2005.

26. Vallerani, E. A review of supersonic sphere drag from the continuum to the free molecular flow regime. In Proceedings of the 41st AGARD (Advisory Group for Aerospace Research and Development) Conference, London, UK, April 1973; pp. 1-15.

27. Moss, J.N. Rarefied flows of planetary entry capsules. In Proceedings of the Special Course on Capsule Aerothermodynamics, von Kármán Institute for Fluid Dynamics (VKI), Rhode-Saint-Genèse, Belgium, 20-22 March 1995.

28. Bird, G.A. Molecular Gas Dynamics and Direct Simulation Monte Carlo; Clarendon Press: Oxford, UK, 1998.

29. Bird, G.A. The DSMC Method, Version 1.1; Amazon: Charleston, SC, USA, 2013.

30. Shen, C. Rarefied Gas Dynamic: Fundamentals, Simulations and Micro Flows; Springer: Berlin, Germany, 2005. 
31. Bird, G.A. Visual DSMC Program for Two-Dimensional Flows, the DS2V Program User's Guide; Version 4.5; G.A.B. Consulting Pty Ltd.: Sidney, Australia, 2008.

32. Bouilly, J.M. Thermal protection of the Huygens Probe during Titan entry: Last questions. In Proceedings of the 2nd International Planetary Probe Workshop, Mountain View, CA, USA, 23-27 August 2004; pp. 113-120.

33. Yelle, R.V.; Strobell, D.F.; Lellouch, E.; Gautier, D. Engineering Models for Titan's Atmosphere. In Proceedings of the ESA Conference on "Huygens: Science, Payload and Mission", Noordwijk, The Netherland, June 1997; pp. 243-256.

(C) 2020 by the authors. Licensee MDPI, Basel, Switzerland. This article is an open access article distributed under the terms and conditions of the Creative Commons Attribution (CC BY) license (http://creativecommons.org/licenses/by/4.0/). 